\title{
Observations of Wood Cell Walls with a Scanning Probe Microscope
}

\author{
Manami Yamashita, Masato Yoshida*, Miyuki Matsuo, Saori Sato, Hiroyuki Yamamoto \\ Graduate School of Bio-agricultural Science, Nagoya University, Nagoya, Japan \\ Email: *yoshida@agr.nagoya-u.ac.jp
}

How to cite this paper: Yamashita, M., Yoshida, M., Matsuo, M., Sato, S. and Yamamoto, H. (2016) Observations of Wood Cell Walls with a Scanning Probe Microscope. Materials Sciences and Applications, 7, 644-653.

http://dx.doi.org/10.4236/msa.2016.710052

Received: September 16, 2016

Accepted: October 17, 2016

Published: October 20, 2016

Copyright $\odot 2016$ by authors and Scientific Research Publishing Inc. This work is licensed under the Creative Commons Attribution International License (CC BY 4.0).

http://creativecommons.org/licenses/by/4.0/

(c) (i) Open Access

\begin{abstract}
Scanning Probe Microscopes (SPMs) observe specimen surfaces with probes by detecting the physical amount of a material between the cantilever and the surface. SPMs have a high resolution and can measure mechanical characteristics such as stiffness, adsorptive properties, and viscoelasticity. These features make it easy to identify the surface structure of complex materials; therefore, the use of SPMs has increased in recent years. Wood cell walls are primarily composed of cellulose, hemicellulose, and lignin. It is believed that hemicellulose and lignin surround the cellulose framework; however, their detailed formation remains unknown. Therefore, we observed wood cell walls via scanning probe microscopy to try to reveal the formation of the cellulose framework. We determined that the size of the cellulose microfibril bundle and hemicellulose lignin module composite was $18.48 \mathrm{~nm}$ based on topography and that the size of the cellulose microfibril bundle was $15.33 \mathrm{~nm}$ based on phase images. In the viscoelasticity image, we found that the viscoelasticities of each cell wall of the same cell were not the same. This is because the cellulose microfibrils in each cell wall lean in different directions. The angle between the leaning of the cellulose microfibril and the cantilever affects the viscoelasticity measurement.
\end{abstract}

\section{Keywords}

Scanning Probe Microscope, Wood Cell Wall, Cellulose Microfibril, Hemicellulose, Lignin

\section{Introduction}

Scanning Probe Microscope (SPM) is a collective term for scanning-type microscopes that do not have a lens for measurement such as Atomic Force Microscopes (AFMs), Scanning Tunneling Microscopes (STMs), and Scanning Near-Field Optical Microscopes (SNOMs). SPMs measure specimen surfaces by detecting the physical amount of 
material between a sharp tip mounted on a cantilever and the surface. SPMs have a higher resolution than scanning electron microscopes, transmission electron microscopes, and optical microscopes. SPMs can have a vertical resolution of $0.01 \mathrm{~nm}$ and a horizontal resolution of $0.2 \mathrm{~nm}$. In addition, they can measure various types of specimens, e.g., dried, wet, conductive, or insulating, in various conditions, e.g., in atmospheric conditions, under water, or in a vacuum. Importantly, SPMs can measure not only topography but also mechanical characteristics, such as stiffness, adsorptive properties, and viscoelasticity, at the same time. These features of SPMs enable the characterization of a surface at a high resolution. Therefore, the application of SPMs for observations of some types of materials, such as metal, ceramic, and biological objects, has been increasing in recent years [1] [2] [3] [4].

Wood is composed of two types of cells: one aligned in the direction of the tree stem axis and the other aligned in the direction of the radius of the stem (Figure 1(a)). Tracheids are one of the former cell types and are the primary elements composing conifers. They are fusiform-shaped, that is, the length of their axial direction is approximately $3 \mathrm{~mm}$ and their width is $30-50 \mu \mathrm{m}$. Tracheids play a role in conducting tissues and provide support. The shape of the cross-section of a tracheid is nearly square, and we refer to the cell walls in the radial direction as the radial walls and those in the tangential direction as the tangential walls (Figure 1(b)). Tracheid cell walls are composed of a primary wall and a secondary wall, and each cell is adhered by a middle lamella. The primary wall is formed at the surface layer of a cell when the cell is elongating and enlarging, and the secondary wall is formed interior to the primary wall after the cell has finished enlarging. The secondary wall has a specific composition for wood. It is composed of a tough microfibril framework and an amorphous substance that surrounds the microfibril. The cellulose microfibril aggregation is composed of several bunched cellulose molecules, and its cross-sectional size is thought to be $16-20 \mathrm{~nm}$ [5]. As shown in Figure 1(c), the secondary wall is divided into layers, S1 (the outer layer), S2 (the middle layer), and S3 (the inner layer), and the orientation of the cellulose microfibril in each layer is different [6]. The tilting directions of the cellulose microfibrils are also different. The orientation of S1 and S3 is an s-helix (a left-hand screw), while the orientation of S2 is a $\mathrm{z}$-helix (a right-hand screw). The microfibril angle is also different; the angle of S2 is $5^{\circ}-30^{\circ}$ while those of S1 and S3 are larger. In addition, S2 is much thicker than other walls and occupies $70 \%-80 \%$ of the cell wall. Therefore, S2 primarily determines the mechanical properties of the wood.

Wood cell walls are composed of cellulose, hemicellulose, and lignin [7]. In simple terms, the structure of a wood cell wall resembles reinforced concrete, with cellulose, lignin, and hemicellulose playing the roles of steel rods, concrete, and wire to strap together the steel rods and concrete, respectively [8]. However, their exact formation in the cell wall remains uncertain. Fahlén and Salmén [9] and Ruel and Goring [10] have stated that the S2 layer consists of concentric lamella, and Sell and Zimmermann [11] have demonstrated the radial formation of the cellulose microfibril and the matrix substance. Conversely, Zimmermann et al. [12] have shown that the cell wall components are randomly arranged in the cell wall. 


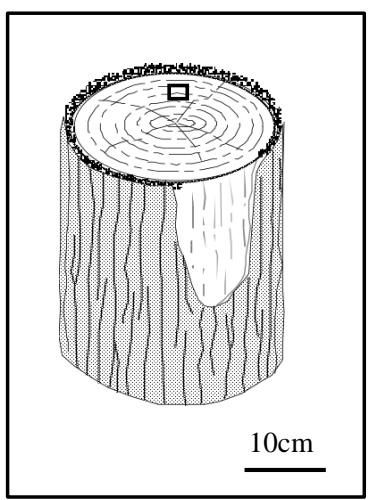

(a)

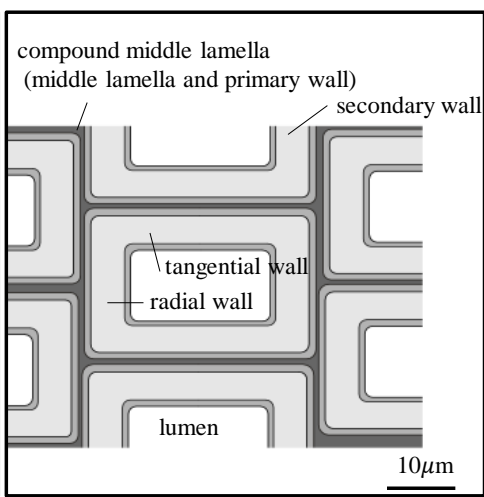

(b)

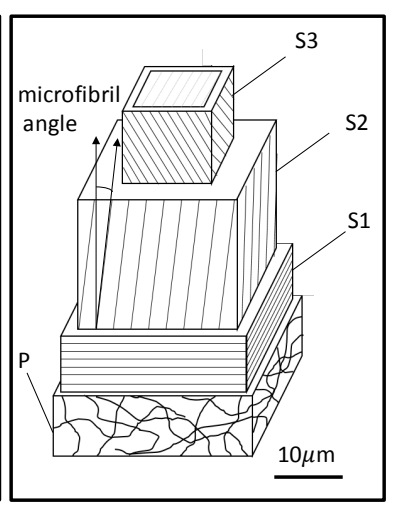

(c)

Figure 1. Schematic model of the hierarchical structure of tracheids. (a) A log; (b) Cross-section of a tracheid that is an enlargement of the area indicated by the black square in (a). A cell wall is composed of a primary wall and a secondary wall, and the cells are adhered by a middle lamella. Primary wall is so thin; therefore, it is difficult to dived compound middle lamella into middle lamella and primary wall. The cell walls in the radial direction are called the radial walls and those in the tangential direction are called the tangential walls. Inside the cell wall, there is lumen; (c) Multilayered tracheid. The secondary cell wall is divided into layers, S1 (the outer layer), S2 (the middle layer), and S3 (the inner layer), and the cellulose microfibrils in each layer are variously oriented. The microfibril orientation of S1 and S3 is an s-helix (a left-hand screw), that of $\mathrm{S} 2$ is a $\mathrm{z}$-helix (a right-hand screw), and that of $\mathrm{P}$ (the primary wall) is random.

In this study, we used SPM to observe wood cell walls. SPM can simultaneously measure topography and mechanical properties at high resolutions, which makes it easy to identify construction elements. Measurement instruments used in past studies have had the following features. Confocal Raman microscope, which obtains composite information, cannot distinguish the construction elements of a wood cell wall due to its low resolution [13]. SEM and TEM have higher resolutions than Raman microscopes but only in measuring topography; therefore, they cannot distinguish between cellulose microfibril, hemicellulose, and lignin on the basis of their configurations.

We observed the S2 layer of wood cell walls by using SPM with the aim of distinguishing the wood cell wall formation in detail.

\section{Material and Method}

It is desirable that a specimen surface be as smooth as possible for SPM measurements because the vertical resolution of SPM is so high that a small amount of asperity could affect the observations. In particular, in phase imaging, large asperity makes it difficult to observe differences in the mechanical properties. It is also necessary that the exact difference in the mechanical properties of a compositional element of the cell wall be observed.

\subsection{Sample Preparation}

A 19-year-old and a 3-year-old Cryptomeria japonica were obtained from an experimental forest at the Nagoya University. These samples were prepared according to the 
following method. Specimens were cut to a size of $2 \mathrm{~mm} \times 2 \mathrm{~mm} \times 5 \mathrm{~mm}$ using a box-cutter and dehydrated with an ethanol series (50\%, 70\%, 95\%, and 100\%). Then, they were replaced with increasing ratios of propylene oxide; after which, the propylene oxide was replaced with increasing ratios of epoxy resin. After resin polymerization in an oven, the entire resin block with the sample was attached to a sample stage. In the same way, cellulose microcrystalline and Klason lignin were embedded in the epoxy resin to form a standard sample. The samples were trimmed with a razor blade. Then, they were trimmed with a DiATOME (Type: Trim 45) to a cross-sectional size of $1 \mathrm{~mm}$ in the radial direction and $0.5 \mathrm{~mm}$ in the tangential direction. Via a rotary microtome using a SYMKnife (Type: SYM2045), $1 \mu \mathrm{m}$ thick sections were obtained. The sections were placed on silicon wafers and dried in an electrical heater. We observed the dried sample using SPM.

\subsection{SPM Observations}

In this study, we used SPM (HITACHI AFM5000 and AFM5200S), which has two measurement modes: Dynamic Force Microscope (DFM) and Viscoelasticity Atomic Force Microscope (VE-AFM). DFM is more suitable than AFM for observations of specimens that are soft or adsorptive. A resonated cantilever measures the specimen surface in the DFM mode. The oscillation amplitude of a cantilever is sensitive to the distance between the cantilever and the surface. Cantilevers scan the surface by maintaining their distance and a constant oscillation amplitude to obtain images. In this mode, we obtained the topography and phase images. Conversely, in the VE-AFM mode, we obtained the topography and the viscoelastic images. Viscoelastic images are created from the flexural oscillation of the cantilever. The sample is vibrated vertically. Due to the agency of the viscoelasticity between the cantilever and the sample surface, the input signal of the longitudinal vibration added to the samples is split between the flexural oscillation of the cantilever and the sample deformation in the output signal. Therefore, in hard areas, the sample deformation is small and the flexural oscillation of the cantilever is large. In this mode, we can obtain more accurate differences in the hardness because the cantilever directly pushes the specimen (in DFM mode, components such as adsorption and electrostatic forces affect the phase lag).

\section{Results}

\subsection{Topography Measurements}

We obtained the topography in both DFM and VE-AFM modes. The measurement principle for obtaining the height information for both modes is based on the z-axis displacement of the cantilever. As shown in Figure 2(a), the cantilever moves during scanning in the direction of the $\mathrm{z}$-axis along the profile of the surface. The $\mathrm{z}$-axis displacement indicates the height of the specimen's surface. The topography is obtained by retrieving the height information at each measurement point on the sample. The topography color is not the actual color of the specimen surface; instead, it indicates the height information according to the scale variation next to the image. 
Figure 2(b) shows the topography of a cross-section of wood cells. We indicate one cell in the figure with a black dashed line. The epoxy resin (lumen) was darker than the cell. A darker color indicates that an area is lower and a lighter color indicates that an area is higher; therefore, the epoxy resin had a lower relief than the cell. The S2 cell wall image observed at a higher magnification is shown in Figure 2(c), and its 3D image is shown in Figure 2(d). In these images, multiple circular structures were observed, and these structures formed a nub-like structure. We focused our attention on the circular structure indicated by the black arrow. This structure may indicate a cellulose microfibril because the wood cell wall is composed of a material component (cellulose) and an amorphous component (hemicellulose and lignin). We measured the diameters of these structures and found them to be $18.48 \mathrm{~nm}$. Terashima et al. have stated that the diameter of cellulose microfibril bundles consisting of approximately 430 cellulose chains is $12 \pm 3 \mathrm{~nm}$ and that the diameter of a composite of a cellulose microfibril bundle and a hemicellulose lignin module is $18 \pm 1 \mathrm{~nm}$ [14]. On the basis of the cross-sectional size,

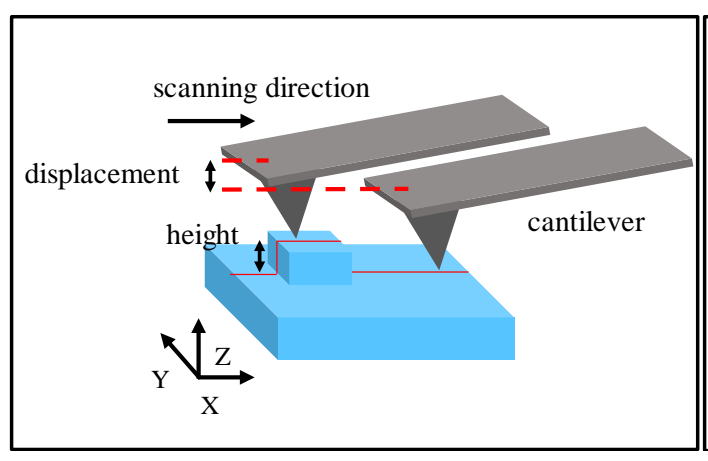

(a)

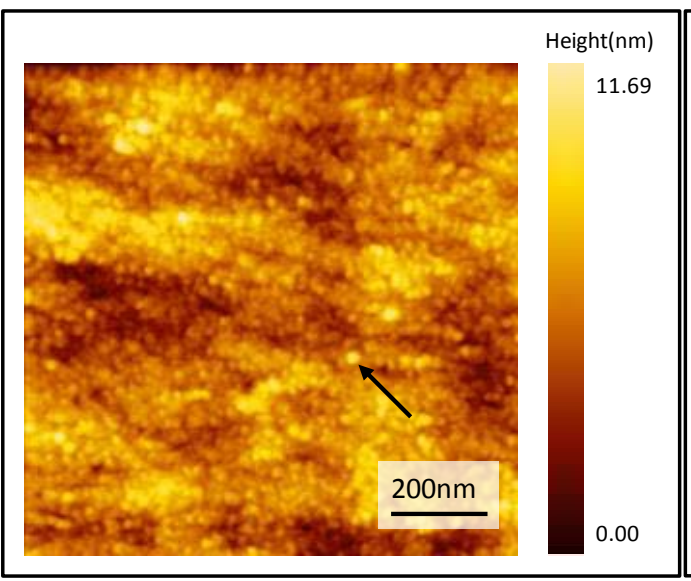

(c)

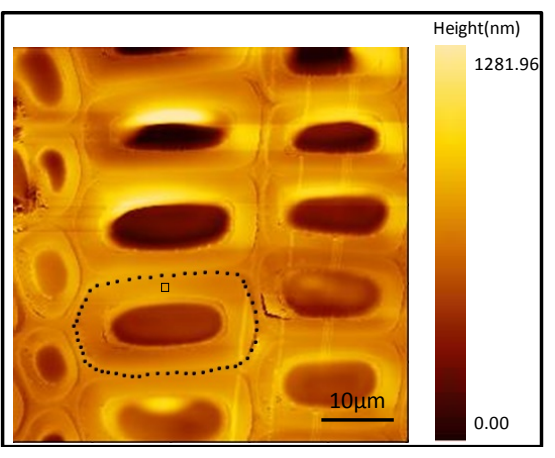

(b)

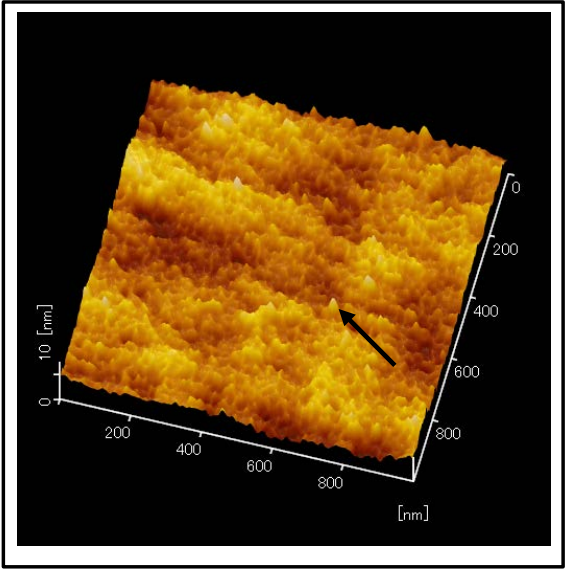

(d)

Figure 2. (a) Schematic of the topography measurement. The cantilever moves in the direction of the $\mathrm{z}$-axis during scanning along the solid red line and the $\mathrm{z}$-axis displacement indicates the height of the specimen's surface; (b) Topography of a wood cell cross-section. The epoxy resin (lumen) is darker than the cell wall indicating depressions; (c) Topography of a cross-section of the S2 layer. This is an enlarged illustration of the area indicated by the black square in (b); (d) A $3 \mathrm{D}$ image of (c). Black arrows indicate the same structure shown in (c). 
we concluded that these structures were composites of cellulose microfibril bundles and hemicellulose lignin modules. That is to say, it is likely that the circular structures are composed of a cellulose axis and surrounding hemicellulose and lignin.

However, this is difficult to prove based on just the shape and size; therefore, we compared the phase images.

\subsection{Phase Measurements}

Phase images are made by detecting the phase lag between the input signal, which is added to the vibrating cantilever, and the output signal, which depends on the mechanical properties of the specimen surface (Figure 3(a)). If the specimen is hard or has low adsorptive properties, the phase shift is small.

Figure 3(b) shows a phase image of the cross-section of a wood cell wall shown in Figure 2(c). The white arrow indicates the same location that was indicated in Figure 2(c) and Figure 2(d), and an enlargement of this structure is shown in Figure 3(c). As shown in Figure 3(c), the color of the circular structure was dark and the substance surrounding it was light. The scale bar next to Figure 3(b) indicates the phase shift. Darker colors indicate that the phase shift is small and lighter colors indicate that the phase shift is large; therefore, the phase shift of the circular structure was smaller than that of the substance around it. We can also observe the cellulose microcrystalline and the Klason lignin embedded in the epoxy resin in the standard sample. Figure 3(d) is composed of two images. As shown in Figure 3(d), the color of the cellulose microcrystalline is darker than that of the Klason lignin. Therefore, the phase shift of the cellulose microcrystalline is smaller than that of the Klason lignin. This means that the circular structure is cellulose and the amorphous substance around the cellulose is hemicellulose and lignin. Next, we measured the size of the circular structures corresponding to the areas thought to be composites of cellulose microfibril bundles and hemicellulose lignin modules in the phase image (Figure 3(b)); the size was $15.33 \mathrm{~nm}$. Therefore, the size measured in the phase image was smaller than that measured using topography. We consider this difference below.

Wood cell walls are composed of cellulose, hemicellulose, and lignin, and it is thought that hemicellulose and lignin surround the cellulose. When cutting the crosssection, circular structures, whose center cores are cellulose, and matrix substances that enclose the cellulose (composites of cellulose microfibril bundles and hemicellulose lignin modules) both appear on the specimen surface as nubs. In the topography image, we measured the sizes of these nubs as circular structures. Conversely, in the phase image, we could distinguish the phase lags of the cellulose and matrix substances, and therefore we measured only the cellulose as the circular structures. That is, the circular structure in the topography indicated composites of cellulose microfibril bundles and hemicellulose lignin modules while that in the phase image indicated cellulose microfibril bundles. Each size is similar to those reported by Terashima et al. [14].

Based on the phase image, we also revealed the spatial pattern of the cellulose and matrix substances in the cross-section of the wood cell wall. 


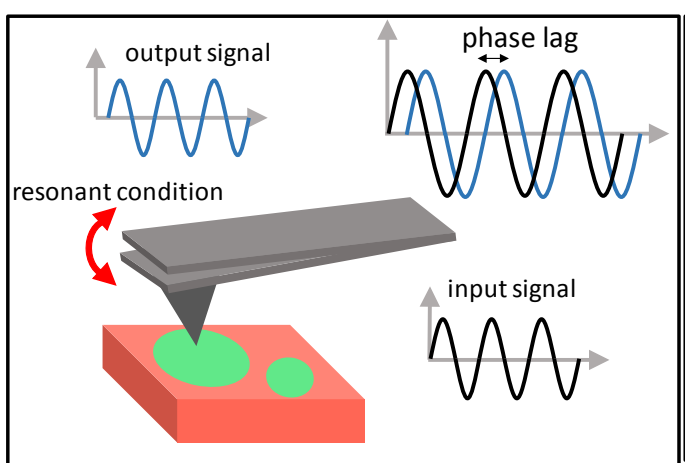

(a)

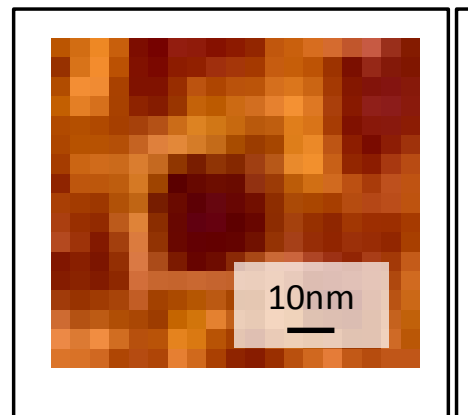

(c)

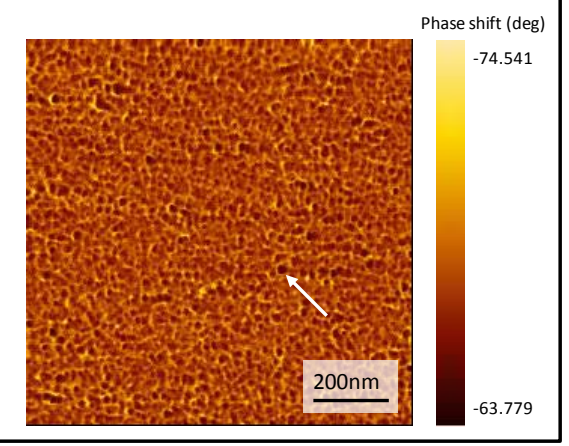

(b)

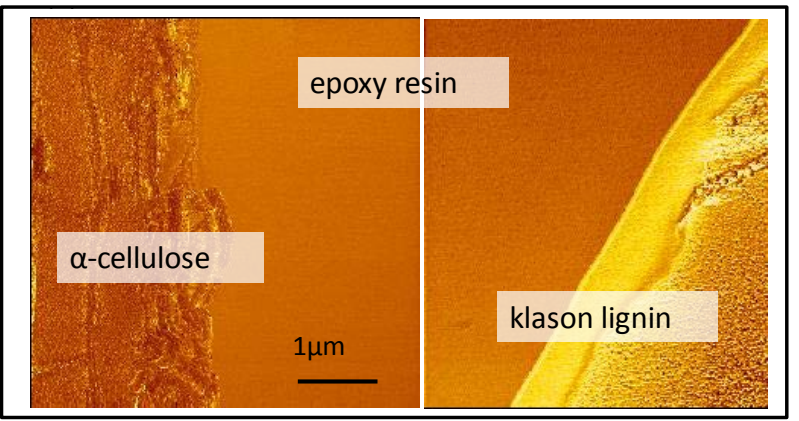

(d)

Figure 3. (a) Schematic of the phase measurement. The phase lag between the input signal and the output signal depends on the mechanical properties of the sample; (b) Phase image of a cross-section of a wood cell wall. This is the same area shown in Figure 1(c). The white arrow indicates the same area indicated in Figure 1(c); (c) Enlargement of the structure pointed to in Figure 2(b). The phase shift of the circular structure is small and that of the amorphous substance around the cellulose is large; (d) Phase image of a-cellulose and Klason lignin. The phase shift of $\alpha$-cellulose is small and that of Klason lignin is large.

\subsection{Viscoelasticity Measurements}

Viscoelastic images are created from the flexural oscillation of the cantilever. This depends on the viscoelastic agency between the cantilever and the sample surface (Figure 4(a)). Figure 4(b) shows a viscoelasticity image of a cross-section of the 3-year-old wood cell. In the viscoelastic image, darker colors indicate soft areas and lighter colors indicate hard areas. Cell walls in the lengthwise direction in this image are the radial walls and those in the cross direction are the tangential walls. This image indicates that the color of the upper tangential wall and the lower tangential wall of the same cell are different. The lower tangential wall was harder than the upper tangential wall. Differences in the viscoelasticity of the same cell were also seen in images reported by Arnould and Arinero [15]. They used the contact resonance mode of an AFM for their observations.

We hypothesized that the differences in the viscoelasticity in the tangential walls were due to the $\mathrm{z}$-helical structure of the cellulose microfibril. The z-helical structure causes the tilt of the cellulose microfibril in the upper tangential wall to be opposite to that in the lower tangential wall. Conversely, the direction that the cantilever applied its 
load to the specimen (it is not vertical to the specimen surface) was constant. Therefore, the direction of the applied load to the cellulose differs between the upper and lower tangential walls (Figure $4(\mathrm{c})$ ). Therefore, this could cause a difference in the viscoelasticity.

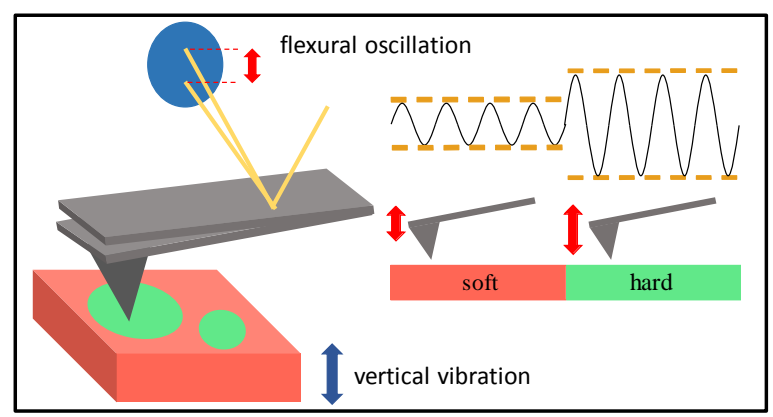

(a)

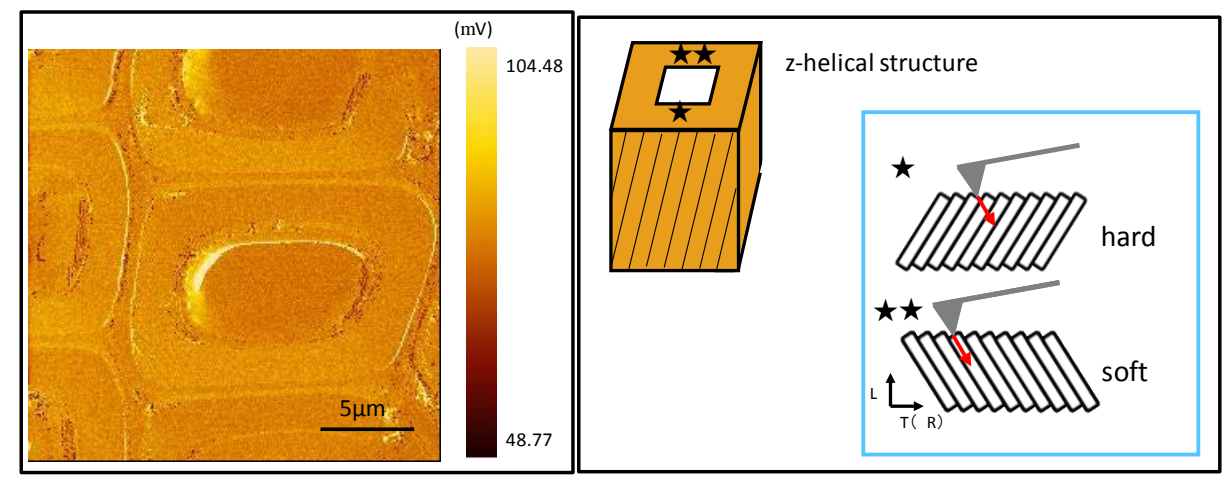

(b)

(c)

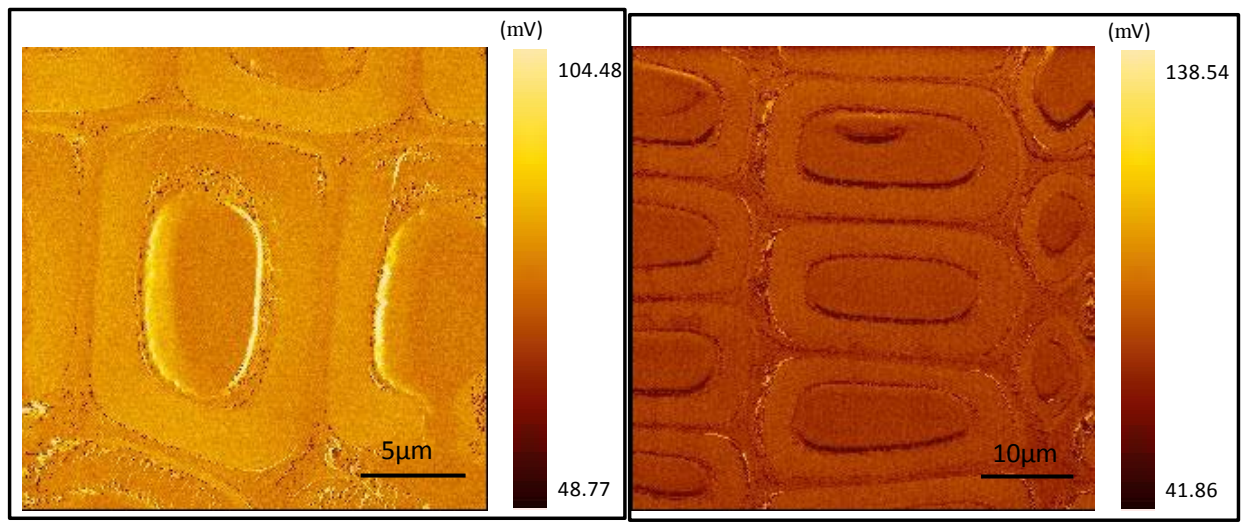

(d)

(e)

Figure 4. (a) Schematic of the viscoelasticity measurement. The oscillation of the cantilever indicates the hardness of the sample; (b) Viscoelasticity image of a cross-section of a 3-year-old wood cell. The viscoelasticities of the tangential walls of the same cell are different; (c) The $z$-helical structure of the secondary cell wall of a wood cell. The cellulose (white line) is tilted; therefore, the direction of the load applied to the cellulose is different in the tangential walls of the same cell; (d) Viscoelasticity image of the same cell in (b) with the specimen rotated $90^{\circ}$ to the right; (e) Viscoelasticity image of a cross-section of a 19-year-old wood cell. There is no difference of viscoelasticities between tangential walls. 
To prove this, we rotated the specimen $90^{\circ}$ to the right and observed it again. By rotating the specimen, the cantilever applied a different load to the specimen not on the tangential walls but on the radial walls. Therefore, we projected that the difference in the viscoelasticity would occur in the radial walls. The result is shown in Figure 4(d). The right and left cell walls are the tangential walls and the upper and lower cell walls are the radial walls. As shown in Figure 4(b), the colors of the tangential walls are the same but those of the radial walls are different. Therefore, the viscoelastic difference is not a characteristic of the tangential wall; rather, it appears due to differences in the direction that the cantilever applies its load to the specimen (i.e., the difference appeared in the tangential walls in Figure 4(b) and in the radial walls in Figure 4(d)).

This viscoelastic difference in the tangential walls was observed only in the 3-yearold wood sample, not in the 19-year-old sample. This is because the 3-year-old sample was juvenile and the microfibril angle of S2 is larger than that in the 19-year-old sample. Subsequently, we need to research how much the tilt affects the viscoelasticity differences and we need to calculate the microfibril angle from the viscoelastic images.

\section{Conclusions}

In this paper, we reported observations of wood cell walls by using a SPM. The ability of SPM to simultaneously observe the topography and mechanical characteristics at high resolutions easily allowed detailed observations of wood cell walls. We found from differences in the mechanical properties of each material that a matrix substance (hemicellulose and lignin) surrounded the cellulose in the cell wall. The phase lag of cellulose was smaller than that of the matrix substance. Regarding the cellulose microfibril size, we found that the cross-sectional size of cellulose microfibril bundles was 15.33 $\mathrm{nm}$ and that composites of cellulose microfibril bundles and hemicellulose lignin modules had a cross-sectional size of $18.44 \mathrm{~nm}$.

In addition, we obtained new information concerning the tilt of the cellulose microfibrils. That is, the viscoelasticity of cell walls of the same cell were different due to the tilting of the cellulose microfibril resulting from the z-helical structure. We can see this difference only when the microfibril angle of the S2 layer is large.

\section{Acknowledgements}

This work was supported by JSPS KAKENHI Grant Number 26292095.

\section{References}

[1] Hummon, M.R., Stollenwerk, A.J. and Narayanamurti, V. (2010) Measuring Charge Trap Occupation and Energy Level in CdSe/ZnS Quantum Dots Using a Scanning Tunneling Microscope. Physical Review B, 81, 115439. http://dx.doi.org/10.1103/PhysRevB.81.115439

[2] Okuzaki, H., Kobayashi, K. and Yan, H. (2009) Thermo-Responsive Nanofiber Mats. Macromolecules, 42, 5916-5918. http://dx.doi.org/10.1021/ma9014356

[3] Myhra, S. (2004) A Review of Enabling Technologies Based on Scanning Probe Microscopy Relevant to Bioanalysis. Biosensors and Bioelectronics, 19, 1345-1354. http://dx.doi.org/10.1016/j.bios.2003.12.016 
[4] Keplinger, T., Konnerth, J., Aguié-Béghin, V., Rüggeberg, M., Gierlinger, N. and Burgert, I. (2014) A Zoom into the Nanoscale Texture of Secondary Cell Walls. Plant Methods, 10, 1. http://dx.doi.org/10.1186/1746-4811-10-1

[5] Salmén, L. and Fahlén, J. (2006) Reflections on the Ultrastructure of Softwood Fibers. Cellulose Chemistry and Technology, 40, 181-185.

[6] Meylan, B.A. and Butterfield, B.G. (1978) Helical Orientation of the Microfibrils in Tracheids, Fibres and Vessels. Wood Science and Technology, 12, 219-222.

[7] Salmén, L. (2004) Micromechanical Understanding of the Cell-Wall Structure. Comptes Rendus Biologies, 327, 873-880.

[8] Zhang, N., Li, S., Xiong, L., Hong, Y. and Chen, Y. (2015) Cellulose-Hemicellulose Interaction in Wood Secondary Cell-Wall. Modelling and Simulation in Materials Science and Engineering, 23, Article Id: 085010.

[9] Fahlén, J. and Salmén, L. (2002) On the Lamellar Structure of the Tracheid Cell Wall. Plant Biology, 4, 339-345. http://dx.doi.org/10.1055/s-2002-32341

[10] Ruel, K.F., Goring, D.A.I. and Barnoud, F. (1978) Lamellation in the S2 Layer of Softwood Tracheids as Demonstrated by Scanning Transmission Electron Microscopy. Wood Science and Technology, 12, 287-291. http://dx.doi.org/10.1007/bf00351930

[11] Sell, J. and Zimmermann, T. (1993) Radial Fibril Agglomerations of the S2 on Transverse-Fracture Surfaces of Tracheids of Tension-Loaded Spruce and White Fir. Holz als Roh-und Werkst., 51, 384.

[12] Zimmermann, T., Thommen, V., Reimann, P. and Hug, H.J. (2006) Ultrastructural Appearance of Embedded and Polished Wood Cell Walls as Revealed by Atomic Force Microscopy. Journal of Structural Biology, 156, 363-369.

http://dx.doi.org/10.1016/j.jsb.2006.06.007

[13] Zhang, Z.H., Ma, J.F., Ji, Z. and Xu, F. (2012) Comparison of Anatomy and Composition Distribution between Normal and Compression Wood of Pinus bungeana Zucc. Revealed by Microscopic imaging Techniques. Microscopy and Microanalysis, 18, 1459-1466. http://dx.doi.org/10.1017/S1431927612013451

[14] Terashima, N., Kitano, K., Kojima, M., Yoshida, M., Yamamoto, H. and Westermark, U. (2009) Nanostructural Assembly of Cellulose, Hemicellulose, and Lignin in the Middle Layer of Secondary Wall of Ginkgo Tracheid. Journal of Wood Science, 55, 409-416. http://dx.doi.org/10.1007/s10086-009-1049-x

[15] Arnould, O. and Arinero, R. (2015) Towards a Better Understanding of Wood Cell Wall Characterisation with Contact Resonance Atomic Force Microscopy. Composites Part A: Applied Science and Manufacturing, 74, 69-76. http://dx.doi.org/10.1016/j.compositesa.2015.03.026 
Submit or recommend next manuscript to SCIRP and we will provide best service for you:

Accepting pre-submission inquiries through Email, Facebook, LinkedIn, Twitter, etc. A wide selection of journals (inclusive of 9 subjects, more than 200 journals)

Providing 24-hour high-quality service

User-friendly online submission system

Fair and swift peer-review system

Efficient typesetting and proofreading procedure

Display of the result of downloads and visits, as well as the number of cited articles

Maximum dissemination of your research work

Submit your manuscript at: http://papersubmission.scirp.org/

Or contact msa@scirp.org 